\title{
Prognostic and clinicopathological value of PD-LI in colorectal cancer: a systematic review and meta-analysis
}

This article was published in the following Dove Press journal: OncoTargets and Therapy

\author{
Lianzhou Yang ${ }^{\prime}$ \\ Rujun Xue $\mathrm{e}^{2,3}$ \\ Chunhua $\operatorname{Pan}^{4}$ \\ 'Radiotherapy Department, Central \\ Hospital of Guangdong Nongken, \\ Zhanjiang, Guangdong, People's Republic \\ of China; ${ }^{2}$ Institute of Dermatology, \\ Guangzhou Medical University, \\ Guangzhou, People's Republic of China; \\ ${ }^{3}$ Department of Dermatology, \\ Guangzhou Institute of Dermatology, \\ Guangzhou, People's Republic of China; \\ ${ }^{4}$ The Ist Ward of the Medical \\ Department, Affiliated Cancer Hospital \& \\ Institute of Guangzhou Medical \\ University, Guangzhou, People's Republic \\ of China
}

Purpose: The prognostic role of programmed death-ligand 1 (PD-L1) in colorectal cancer remains unclear. We employed a meta-analysis to explore the prognostic value of PD-L1 and to ascertain the relationship between PD-L1 expression and clinicopathological characteristics in CRC patients.

Methods: We systematically searched PubMed, Embase and the Cochrane Library until October 2018. Eligible studies about colorectal cancer that pay attention to PD-L1 expression and studies reporting survival information were included. In order to evaluate the prognostic role of PD-L1 for overall survival (OS) and recurrence-free survival (RFS)/disease-free survival (DFS), Hazard ratio (HR) with 95\% confidence interval (CI) was used. Odds ratio (OR) with 95\% CI was selected to appraise the correlation between PD-L1 with clinicopathological characteristics of colorectal cancer patients. Begg's funnel plot was used to assess publication bias.

Results: Twelve studies involving 4344 patients published from 2013 to 2018 were included in this meta-analysis. Pooled results revealed that PD-L1 overexpression was relevant to shorter OS (HR 1.47, 95\% CI $=1.01-2.15, p=0.04$ ) and shorter RFS/DFS (HR 1.47, 95\% $\mathrm{CI}=1.01-2.15, p=0.04)$. Moreover, Patients with high expression of PD-L1 associated with inferior tumor stage $(\mathrm{OR}=0.57,95 \% \mathrm{CI}: 0.45,0.74, p<0.0001)$ and Vascular invasionnegativity $(\mathrm{OR}=0.75,95 \% \mathrm{CI}: 0.6,0.94, p=0.01)$. But the expression of PD-L1 is not related to age, sex, tumor location, tumor differentiation, $\mathrm{pT}$ stage, $\mathrm{pN}$ stage, MSI/MMR status.

Conclusion: This meta-analysis revealed that PD-L1 can serve as a significant biomarker for negative prognosis and the adverse clinicopathological features of colorectal cancer and could facilitate the better management of individual patients.

Keywords: PD-L1, colorectal cancer, prognosis, meta-analysis

\section{Introduction}

Colorectal cancer (CRC) is the most commonly diagnosed cancer worldwide at present, being the third and fifth leading cause of cancer death in the United States and China, respectively. ${ }^{1,2}$ About $39 \%$ of patients were diagnosed with localized stage CRC, for which the 5-year survival rates is $90 \%$. However, the patients diagnosed with the regional and distant-stage disease and the survival rate descend on $71 \%$ and $14 \% .^{3}$ New treatments for primary, regional and metastatic CRC have been developed, including laparoscopic surgery for primary; resection of the liver and lungs metastatic disease; radiotherapy for rectal cancer and some forms of metastatic disease; neoadjuvant and palliative chemotherapy; targeted therapy,
Correspondence: Lianzhou Yang Radiotherapy Department, Central Hospital of Guangdong nongken, No. 2 Renmin Avenue, Xiashan District,

Zhanjiang, Guangdong 524000, People's Republic of China

Tel +86I 37I 9447962

Fax +8607592633646

Email yanglianzhoumail@gmail.com 
immunotherapy, and vaccines. ${ }^{4,5}$ Therefore, it is critical to early detection, the effectiveness of prevention, and management to improving treatment strategies and patient outcomes.

However, some locally advanced and distant metastatic CRC patients lost the chance of surgery, so chemotherapy is basically the only option for advanced and metastatic patients; but these have only modest efficacy and are ineffective against distant metastases. ${ }^{6,7}$ Moreover, these treatments generate side effects that can limit their use. In recent years, more attention has been placed on developing immunotherapies, specially programmed death 1 (PD-1) and programmed death ligand-1 (PD-L1).PD-1/PD-L1 is one of the most important co-stimulatory signals; its-mediated immunosuppression is an important part of the dynamic balance of the immune system because it limits the immune response and prevents autoimmune diseases. The present study found that CD274 (PD-L1, B7-H1) was first cloned in $1999 .{ }^{8}$ The expression profiles of CD274 (PD-L1) in human tumors have been revealed. ${ }^{9,10}$ Hunmantumor-associated APCs include tumor microenvironment dendritic cells (DCs), tumor-draining lymph nodes DCs, ${ }^{11,12}$ macrophages, ${ }^{13,14}$ fibroblasts, ${ }^{15}$ and $\mathrm{T}$ cells ${ }^{16}$ were observed a high level of CD274 (PD-L1) protein expression, except tumor cell. Many studies have found that engagement of PD-L1 with its receptor PD-1 on T cells delivers a signal that inhibits $\mathrm{T}$ cell proliferation, resulting in tumor immune evasion. ${ }^{17}$ PD-1/PD-L1 immune checkpoint inhibitors have been used to treat melanoma, non-small cell lung cancer, renal cell carcinoma, lymphoma, and bladder cancer. ${ }^{18}$ However, the efficacy of immune checkpoint inhibitors for the treatment of CRC is limited. Different research has studied the PD-L1 expression of the prognosis role of CRC; nevertheless, the results were not consistent. Some investigations showed that overexpression of PD-L1 forecasted poor survival in CRC, ${ }^{19-24}$ but other investigations presented negative results. $^{25,26}$

To tackle this problem, we employed meta-analysis to synthetic estimate the value of PD-L1 as a prognostic biomarker, and to clarify the relationship between PD-L1 expression and clinicopathological characteristic in CRC patients.

\section{Materials and methods}

This meta-analysis is based on the Preferred Reporting Items for Systematic Reviews and Meta-analysis (PRISMA) guidelines. ${ }^{30}$ Our research was based on data from previously published studies; thus ethical ratify was unnecessary.

\section{Search strategy}

The Cochrane Library, Embase and PubMed were systematically searched. The strategy used was to search for the following words in relevant literature: ("Colorectal Neoplasms" OR "Neoplasms, Colorectal" OR "Colorectal Neoplasm" OR "Neoplasm, Colorectal" OR "Colorectal Tumors" OR "Colorectal Tumor" OR "Tumor, Colorectal" OR "Tumors, Colorectal" OR"Colorectal Carcinoma" OR "Carcinoma, Colorectal" OR "Carcinomas, Colorectal" OR "Colorectal Carcinomas" OR "Colorectal Cancer" OR "Cancer, Colorectal" OR "Cancers, Colorectal" OR "Colorectal Cancers") AND ("CD274" OR "B7-H1" OR "PD-L1" OR "PD-L1” OR “CD274 Antigens” OR "B7-H1 Immune Costimulatory Protein" OR "B7 H1 Immune Costimulatory Protein" OR "B7-H1 Antigen" OR "Antigen, B7-H1" OR "B7 H1 Antigen" OR "PD-L1 Costimulatory Protein" OR "Costimulatory Protein, PDL1" OR "PD L1 Costimulatory Protein" OR "Programmed Cell Death 1 Ligand 1 Protein" OR "CD274 Antigen" OR "Antigen, CD274" OR "Programmed Cell Death 1 Ligand 1" OR "B7H1 Immune Costimulatory Protein"). To recognize more research, we also retrospect the reference lists of relevant articles.

\section{Selection criteria}

The eligible researches were contained in this metaanalysis based on the following criteria: (1) Patients with colorectal cancer confirmed by pathology. (2) Immunohistochemistry (IHC) was used to detect the expression of PD-L1 in colorectal cancer tissue. (3) Studies reported 5-year OS, HR with 95\% confidence interval $(95 \% \mathrm{CIs})$, or reported original survival curves. (4) Their full texts were available. This analysis exclude articles based on the following standards: (1) non-English; (2) animal experiments; (3) comment, letters, review or case reports; (4) deficiency data to report the risk ratios (RR) and $95 \%$ confidence interval $(95 \% \mathrm{CI})$, or the Kaplan-Meier curve could not be extracted. When duplicate publications were identified, only the most complete or most recent article was included.

\section{Data extraction}

All relevant articles data were extracted by two independent reviewers (Lianzhou Yang, Rujun Xue). The information was extracted from each study included: first author, country, date of publication, number of patients, duration of follow up, age, histological type of tumor, tumor site, 
grade at diagnosis, number of patients with PD-L1 positive, cut-off value, antibody, survival data, Kaplan-Meier curves. ALL divergences were settled by discussion and the achievement of consensus.

\section{Quality assessment}

According to the Newcastle-Ottawa Scale (NOS), two researchers independently assessed each study for quality. (Lianzhou Yang, Rujun Xue). The NOS maximum possible score is 9 points. Each study included was judged on three perspectives: (I) the selection (representativeness, selection of the non-exposed, ascertainment of exposure and outcome of interest); (II) the comparability; and (III) the ascertainment of either the exposure or outcome of interest (assessment and follow-up). A study that received a score of 6 or higher was considered the high-quality study.

\section{Statistical analysis}

The software used in this work was Review Manager Software, Version 5.3 and Stata version12.0. The hazard ratio (HR) with $95 \%$ CI was utilized to assess the relationship between the expression of PD-L1 and OS, DFS, and RFS. The merged ORs and the 95\% CIs were utilized to quantitatively determine the relationship between PD-L1 and clinicopathological feature of the patient. The heterogeneity between included studies was examined using $\mathrm{I}^{2}$ and Q text. If $p<0.1$ or $\mathrm{I}^{2}>50 \%$, which demonstrated significant heterogeneity, the fixed effect model was used for meta-analysis. If there was significant heterogeneity, the random model was used. In this analysis, $p$-values $<0.05$ were considered statistically significant.

\section{Results}

\section{Search results}

After the primary retrieval, 675 potentially relevant articles were initially identified with three databases searched. After removing 156 duplicates, still 519 studies were identified for the title/abstract; however, 422 manuscripts were excluded for the following reasons: not original papers (eg, case report, review, and meta-analysis), animal studies, not colorectal cancer-related studies, not PD-L1related studies, irrelevant, or were not published in English. Subsequently, 95 articles remained for further full-text assessment. Thereafter, 36 articles were excluded due to insufficient data, 2 articles lack of survival outcomes, 13 were no-original articles and 7 were unfinished clinical trials. Ultimately, twelve studies ${ }^{19-26,31-34}$ published from 2013 to 2018 were included in this metaanalysis (Figure 1).

\section{Characteristics of included studies}

Twelve studies involving 4344 patients published from 2013 to 2018 were included in this meta-analysis. The number of patients in each study arranged from 90 to 1420. The immunochemical staining (IHC) was used to confirm PD-L1 expression oftumor tissues in all studies. Three pieces of research were performed in Western countries and nine kinds of research were implemented in Asian countries. The quality of the selected researches wasassessed by using the Newcastle-Ottawa Scale and found to range from 6 to 8 , indicating that the studies were of high quality. Table 1 describes the detailed characteristics of the twelve studies.

\section{Correlation between PD-LI expression and survival}

We evaluated the prognostic role of PD-L1 expression of OS, RFS, and DFS. Eight researches $(n=3198)$ reported OS, as significant heterogeneity existed (Cochran's Q, $\left.p<0.00001, \mathrm{I}^{2}=88 \%\right)$.Pooled result by random model revealed high expression of PD-L1 was correlated with poor OS than the absence of PD-L1 expression (HR 1.47, $95 \% \mathrm{CI}=1.01-2.15, p=0.04$ ) (Figure 2A). Because heterogeneity existed, subgroup analysis was performed. Subgroup analysis based on different analytical methods, integrated HR for OS was 1.29 (95\% CI: $0.90-1.86$, $p=0.16$ ) (Figure 2B). Elucidating the effect of different cut-off of PD-L1 expression of the results, we performed subgroup analysis stratified by cut-off. When score $<4$ or cut-off $\leq 5 \%$ the heterogeneity was negligible, but there was no statistical significance in all subgroup between PDL1 expression and overall survival (Figure 3A).

Seven $(\mathrm{n}=1697)$ reported RFS/DFS. As significant heterogeneity existed (Cochran's Q, $p<0.0001, \mathrm{I}^{2}=80 \%$ ). Pooled result by random model revealed PD-L1 overexpression was associated with shorter RFS/DFS than the absence of PD-L1 expression (HR 1.47, 95\% $\mathrm{CI}=1.01-2.15, p=0.04)$ (Figure 2C). Because heterogeneity existed, subgroup analysis was performed. Subgroup analysis based on different analytical methods, integrated HR for RFS/DFS was 1.86 (95\% CI: $1.10-3.14, p=0.02$ ) (Figure 2D). Elucidating the effect of different cut-off of PD-L1 expression of the results, we performed subgroup analysis stratified by cut-off. When cut-off score $<4$ or 


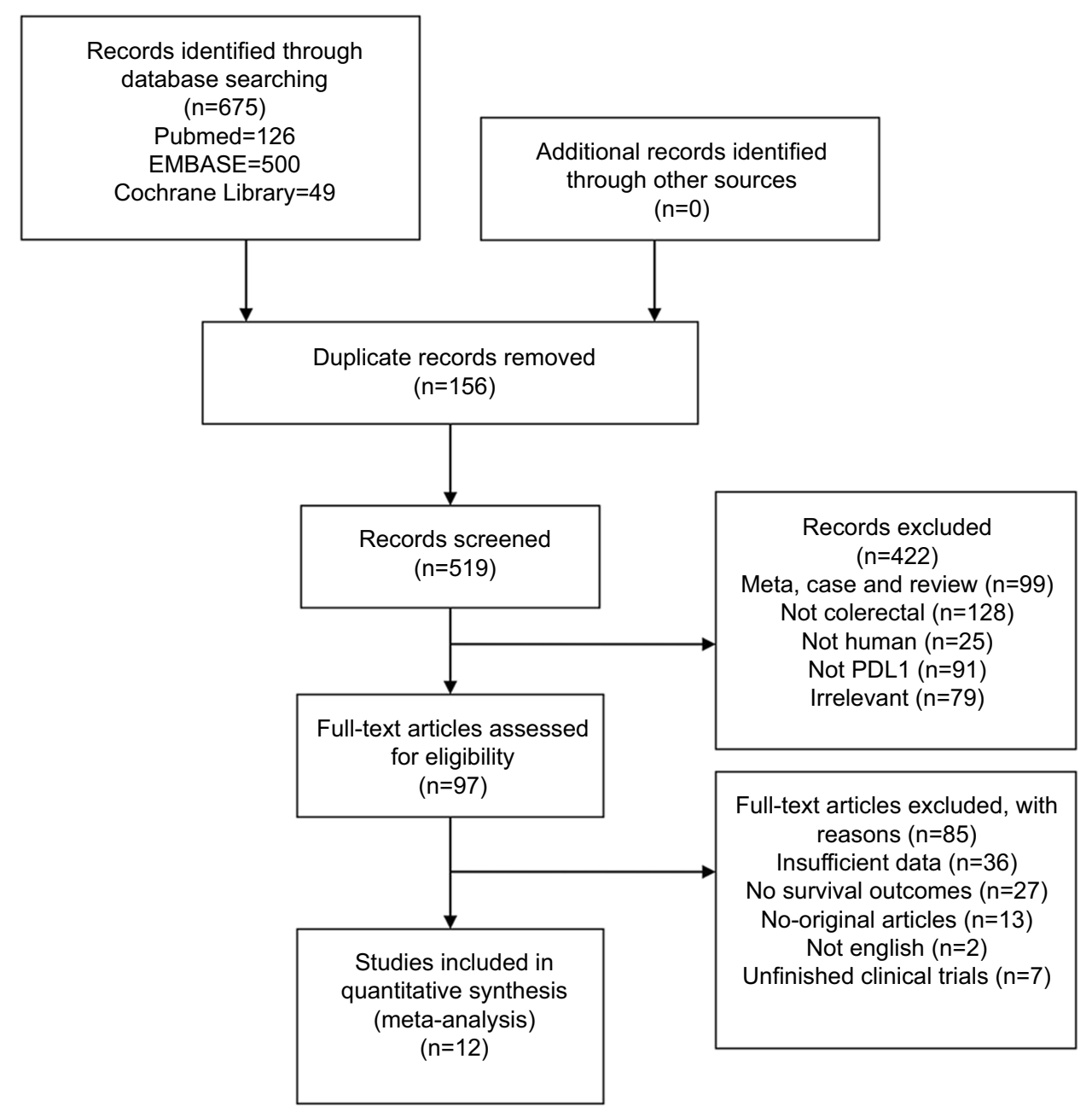

Figure I Flow chart of the literature search and study selection protocols.

$\leq 5 \%$ the heterogeneity was negligible. There was no statistical significance in cut-off $>5 \%$ or score $>4$ subgroups between PD-L1 expression and RFS/DFS (Figure 3B).

\section{Correlation between PD-LI expression and tumor clinicopathlogical parameters}

Moreover, we investigated the relationship between PD-L1 expression and clinicopathological parameters, including sex, age, tumor location, tumor differentiation, tumor stage, $\mathrm{pT}$ stage, $\mathrm{pN}$ stage, vascular invasion, MSI/MMR status. The analysis of each parameter includes at least three studies. The merged results demonstrated that PD-L1 expression suggests in patients with poorer tumor stage ( $\mathrm{OR}=0.57,95 \% \mathrm{CI}$ : $0.45,0.74, p<0.0001)$ and Vascular invasion-negativity (OR $=0.75,95 \% \mathrm{CI}: 0.6,0.94, p=0.01$ ) (Figure 4). However, there was no association between PD-L1 expression and age $(\mathrm{OR}=0.95,95 \% \mathrm{CI}: 0.73,1.22, \mathrm{p}=0.67)$, sex ( $\mathrm{OR}=0.97,95 \%$ CI: $0.84,1.13, p=0.72)$, tumor location $(\mathrm{OR}=1.12,95 \% \mathrm{CI}$ :
$0.87,1.45, p=0.37)$, tumor differentiation ( $\mathrm{OR}=1.18,95 \% \mathrm{CI}$ : $0.94,1.48, p=0.15$ ), pT stage ( $\mathrm{OR}=0.81,95 \% \mathrm{CI}: 0.44,1.48$, $p=0.49)$, $\mathrm{pN}$ stage ( $\mathrm{OR}=0.72,95 \% \mathrm{CI}: 0.44,1.17, p=0.18)$, MSI/MMR status (OR $=0.54,95 \% \mathrm{CI}: 0.16,1.90, p=0.34)$ (Figure S1).

\section{Sensitivity analyses}

We delete any single study at a time individually, which to assess the stability of the results. The results show that there is no separate study significantly affected the total HRs, this meta-analysis of the results are credible.

\section{Publication bias}

The funnel plot did not indicate any evidence of publication bias (Figure 5).

\section{Discussion}

The PD-1/PD-L1 pathway has become a promising therapeutic target for various human malignancies, including 


\begin{tabular}{|c|c|c|c|c|c|c|c|c|c|}
\hline 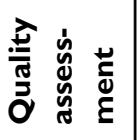 & $\infty$ & $\infty$ & 0 & $0 \wedge 0$ & 05 & ○ & $\infty$ & $\wedge$ & $\infty \wedge$ \\
\hline 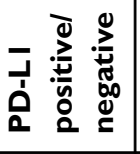 & $\frac{m}{\frac{m}{a}}$ & 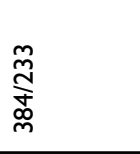 & $\frac{0}{\frac{O}{a}}$ & 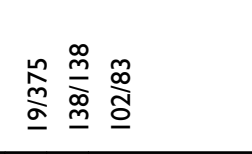 & $\stackrel{m}{\square}$ & ఫ & ' & 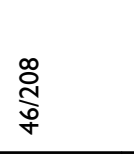 & 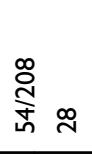 \\
\hline 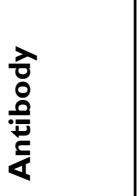 & 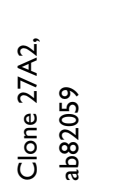 & 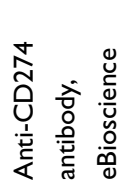 & $\frac{z}{0}$ & 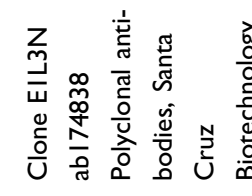 & 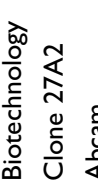 & 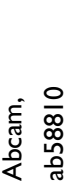 & 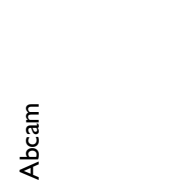 & $\frac{z}{\frac{z}{n}}$ & 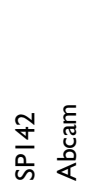 \\
\hline & 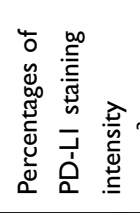 & & ํํㅅ & 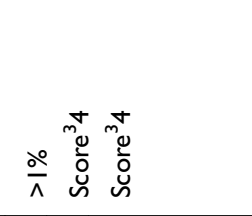 & 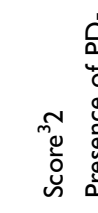 & 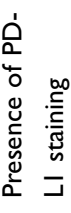 & 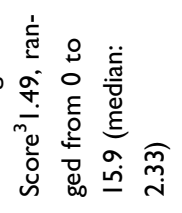 & 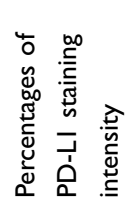 & 苍 \\
\hline 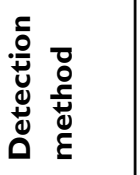 & $\stackrel{\cup}{\underline{I}}$ & $\stackrel{U}{\underline{\underline{I}}}$ & $\stackrel{\cup}{\underline{I}}$ & $\stackrel{U}{\underline{\underline{I}}} \underset{\underline{\underline{I}}}{\underline{U}} \underline{\underline{\underline{I}}}$ & $\stackrel{u}{\underline{I}}$ & $\underline{\underline{u}}$ & $\stackrel{U}{\underline{I}}$ & $\stackrel{\cup}{\underline{I}}$ & $\underline{\underline{\underline{I}}} \underline{\underline{\underline{I}}}$ \\
\hline 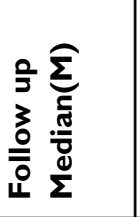 & ' & 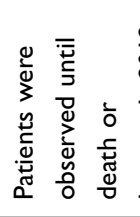 & 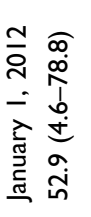 & 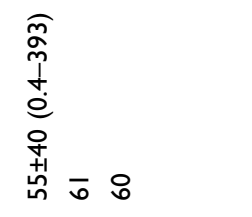 & 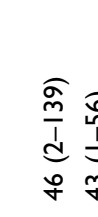 & & ત্ড & $\begin{array}{l}\widehat{0} \\
o \\
\frac{1}{d} \\
\mathcal{q}\end{array}$ & 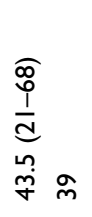 \\
\hline 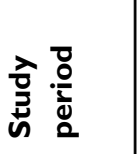 & $\begin{array}{l}\frac{0}{\sigma} \\
\frac{1}{\infty} \\
\frac{\infty}{0}\end{array}$ & 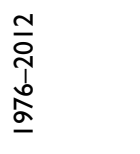 & $\begin{array}{l}\text { No } \\
\text { ஸे } \\
\text { ò }\end{array}$ & . & 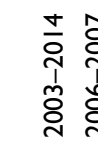 & & 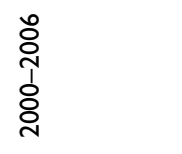 & $\begin{array}{l}\text { Oे } \\
\text { 1 } \\
\text { ò } \\
\text { }\end{array}$ & 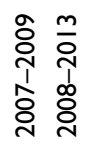 \\
\hline 总 & $\begin{array}{l}\widehat{o} \\
0 \\
0 \\
0 \\
\sigma \\
0 \\
0\end{array}$ & $\begin{array}{l}\infty \\
0 \\
0 \\
+1 \\
0 \\
0 \\
0 \\
0\end{array}$ & 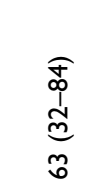 & 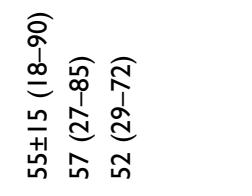 & 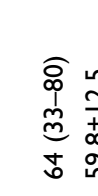 & & นิ & 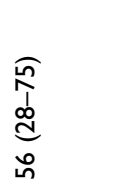 & 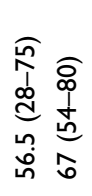 \\
\hline 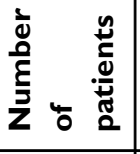 & 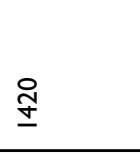 & $\frac{1}{6}$ & $\stackrel{\mathscr{\sim}}{\sim}$ & 只 $\stackrel{\sim}{\sim} \varrho$ & $8 \stackrel{9}{\square}$ & $\underline{\tilde{F}}$ & 今े & 岕 & ํㅗ 오 \\
\hline 胥 & 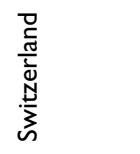 & 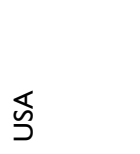 & 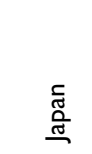 & 芯莺 & 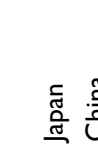 & 胥 & 胥 & 突 & 疍 \\
\hline ঠ & $\bar{m}$ & $\overline{\grave{N}}$ & $\overline{\bar{N}}$ & 유 윰 & $\stackrel{\circ}{\frac{m}{c}}$ & & $\frac{m}{i}$ & $\bar{i}$ & 윰 \\
\hline 六 & 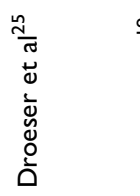 & 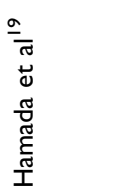 & 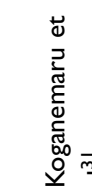 & 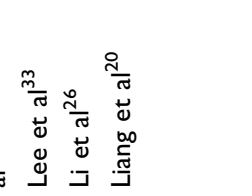 & 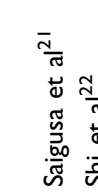 & 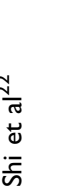 & $\begin{array}{l}\frac{\pi}{\sigma} \\
\bar{\sigma} \\
0 \\
00 \\
0 \\
0\end{array}$ & 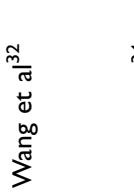 & 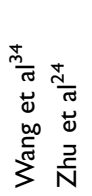 \\
\hline
\end{tabular}




\begin{tabular}{|c|c|c|c|c|}
\hline $\begin{array}{l}\text { A } \\
\text { Study or subgroup }\end{array}$ & log[Hazard ratio] & SE & Weight & $\begin{array}{c}\text { Hazard Ratio } \\
\text { IV, Random, } 95 \% \mathrm{CI}\end{array}$ \\
\hline Droeser RA 2013 & -0.16 & 0.02 & $15.9 \%$ & $0.85[0.82,0.89]$ \\
\hline Hamada T 2017 & 0.29 & 0.18 & $14.0 \%$ & $1.34[0.94,1.90]$ \\
\hline Li Y 2016 & -0.5 & 0.23 & $13.0 \%$ & $0.61[0.39,0.95]$ \\
\hline Liang M 2014 & 0.55 & 0.21 & $13.0 \%$ & $1.73[1.15,2.62]$ \\
\hline Saigusa S 2016 & 0.82 & 0.4 & $9.4 \%$ & $2.27[1.04,4.97]$ \\
\hline Shi SJ 2013 & 0.96 & 0.32 & $11.1 \%$ & $2.61[1.39,4.89]$ \\
\hline Song M 2013 & 0.73 & 0.22 & $13.2 \%$ & $2.08[1.35,3.19]$ \\
\hline zhu H 2015 & 0.83 & 0.36 & $10.2 \%$ & $2.29[1.13,4.64]$ \\
\hline Total $(95 \% \mathrm{Cl})$ & & & $100.0 \%$ & $1.47[1.01,2.15]$ \\
\hline
\end{tabular}

\begin{tabular}{|c|c|c|c|c|}
\hline $\begin{array}{l}\text { B } \\
\text { Study or Subgroup }\end{array}$ & $\log [$ Hazard ratio] & SE & Weight & $\begin{array}{c}\text { Hazard Ratio } \\
\text { IV, Random, } 95 \% \mathrm{CI}\end{array}$ \\
\hline Droeser RA 2013 & -0.08 & 0.02 & $25.0 \%$ & $0.92[0.89,0.96]$ \\
\hline Hamada T2017 & -0.18 & 0.17 & $20.7 \%$ & $1.20[0.86,1.67]$ \\
\hline Li Y 2016 & 0.05 & 0.25 & $17.2 \%$ & $1.05[0.64,1.72]$ \\
\hline Saigusa S 2016 & 0.9 & 0.41 & $11.2 \%$ & $2.46[1.10,5.49]$ \\
\hline Shi SJ 2013 & 1.02 & 0.27 & $16.3 \%$ & $2.77[1.63,4.71]$ \\
\hline zhu H 2015 & -0.37 & 0.47 & $9.6 \%$ & $0.69[0.27,1.74]$ \\
\hline Total $(95 \% \mathrm{CI})$ & & & $100.0 \%$ & $1.29[0.90,1.86]$ \\
\hline
\end{tabular}

\section{C}

Study or Subgroup log[Hazard ratio] SE Weight IV, Random, 95\% CI

\begin{tabular}{|c|c|c|c|c|}
\hline Koganemaru S 2017 & 0.86 & 0.34 & $14.4 \%$ & $2.36[1.21,4.60]$ \\
\hline Lee LH 2016 & 1.28 & 0.61 & $9.4 \%$ & $3.60[1.09,11.89]$ \\
\hline Li Y 2016 & -0.58 & 0.21 & $17.0 \%$ & $0.56[0.37,0.85]$ \\
\hline Liang M 2014 & 0.6 & 0.21 & $17.0 \%$ & $1.82[1.21,2.75]$ \\
\hline Saigusa S 2016 & 1.2 & 0.42 & $12.8 \%$ & $3.32[1.46,7.56]$ \\
\hline Wang L 2016 & 0.55 & 0.27 & $15.8 \%$ & $1.73[1.02,2.94]$ \\
\hline Wang L 2017 & 0.33 & 0.38 & $13.6 \%$ & $1.39[0.66,2.93]$ \\
\hline Total $(95 \% \mathrm{Cl})$ & & & $100.0 \%$ & $1.71[1.02,2.86]$ \\
\hline
\end{tabular}

Test for overall effect: $Z=2.03(P=0.04)$
Hazard Ratio IV, Random, $95 \% \mathrm{CI}$

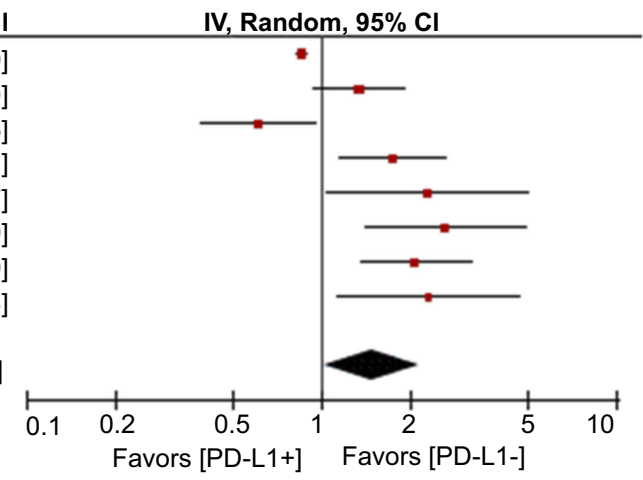
IV, Random, $95 \% \mathrm{Cl}$

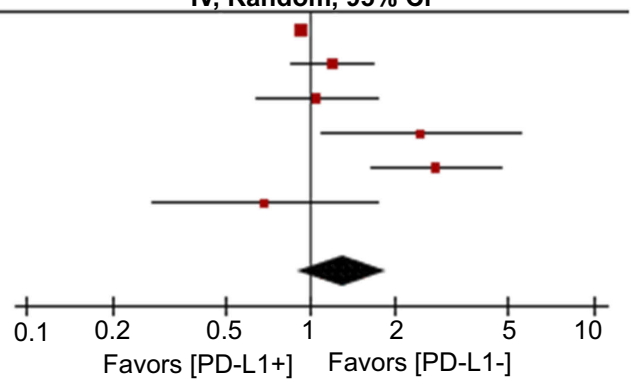

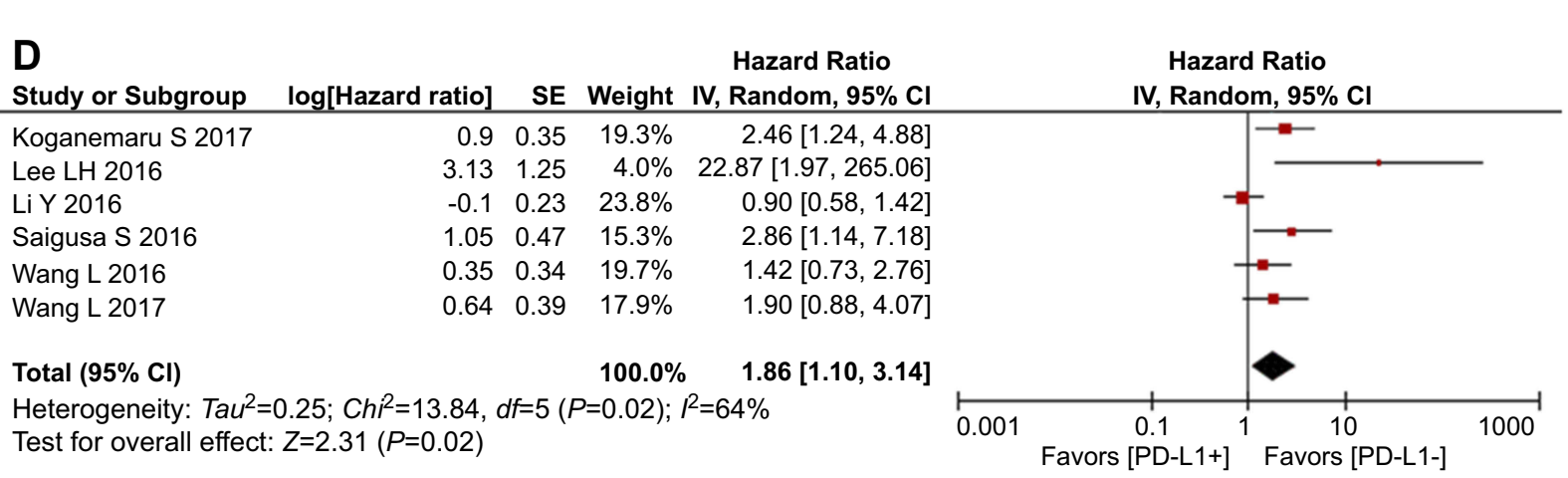

Figure 2 Forest plot describing the association between PD-LI expression and prognosis of patients with CRC patient. (A) OS; (B) OS by multivariate analysis; (C) RFS/ DFS; (D) RFS/DFS by multivariate analysis.

melanoma, non-small-cell lung cancer, head and neck cancer, Hodgkin's lymphoma, gastric cancer, and urothelial cancer. Some clinical trials have shown that PD-1/PD-L1 blockage could benefit prognosis. ${ }^{35-39}$
Nonetheless, there are no consistent results for the prognostic value of PD-L1 in colorectal cancer patients. Lots researches have indicated that the expression of PD-L1 is correlated with an obviously poor survival, ${ }^{19,10-24}$ while 


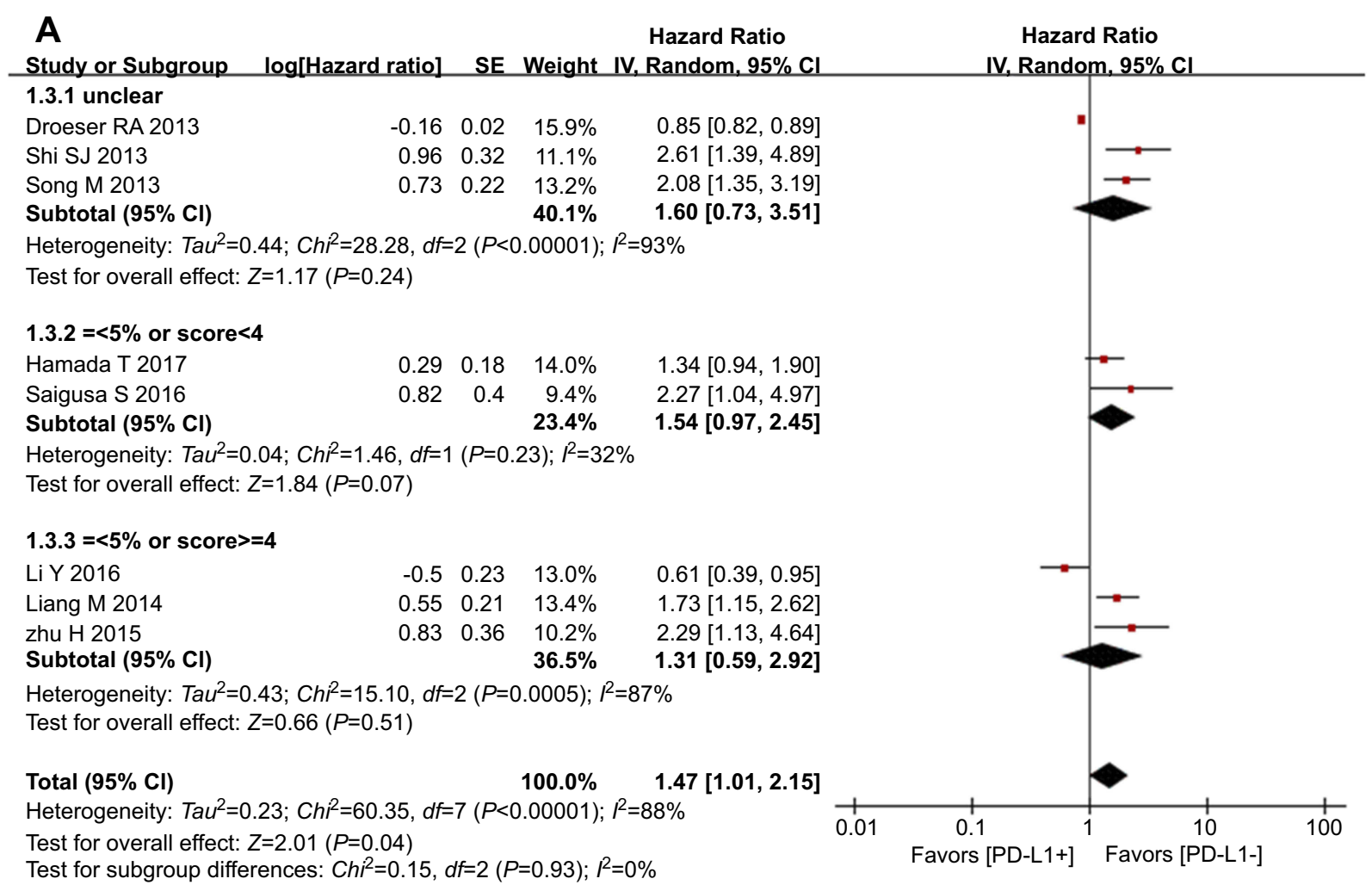

Test for subgroup differences: $C h i^{2}=0.15, d f=2(P=0.93) ; P^{2}=0 \%$

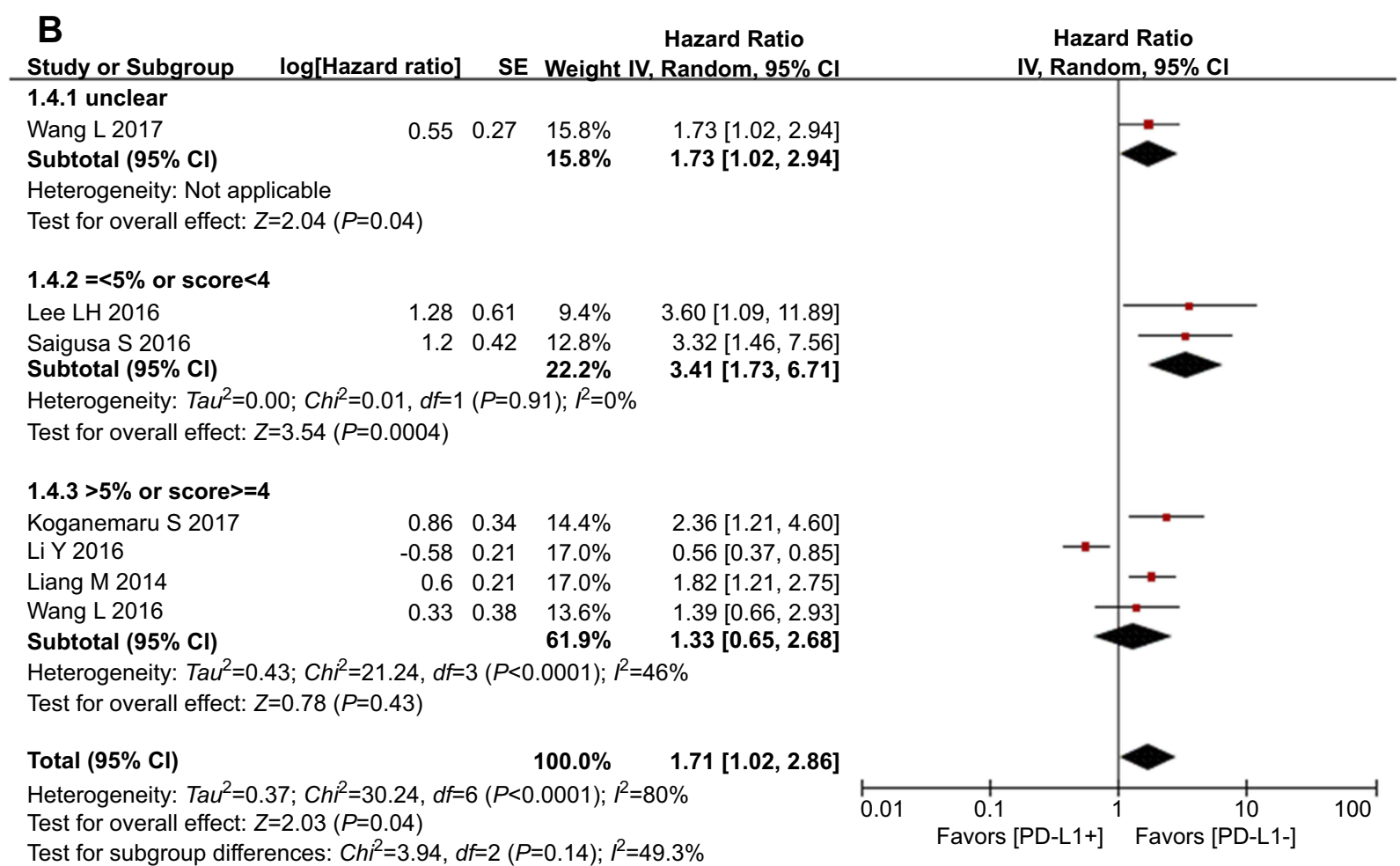

Figure 3 (A) Subgroup analysis based on a different cut-off of association between PD-LI expression and OS. (B) Subgroup analysis based on a different cut-off of association between PD-LI expression and RFS/DFS.

some study accounted the opposite effect. ${ }^{25,26}$ Several studies have revealed the relationship between PD-L1 and Solid Tumors. ${ }^{27-29}$ However, those studies differs from our in that their subjects include all solid tumors in which the number of colorectal cancers included too few, and our study provides an in-depth analysis between PD- 


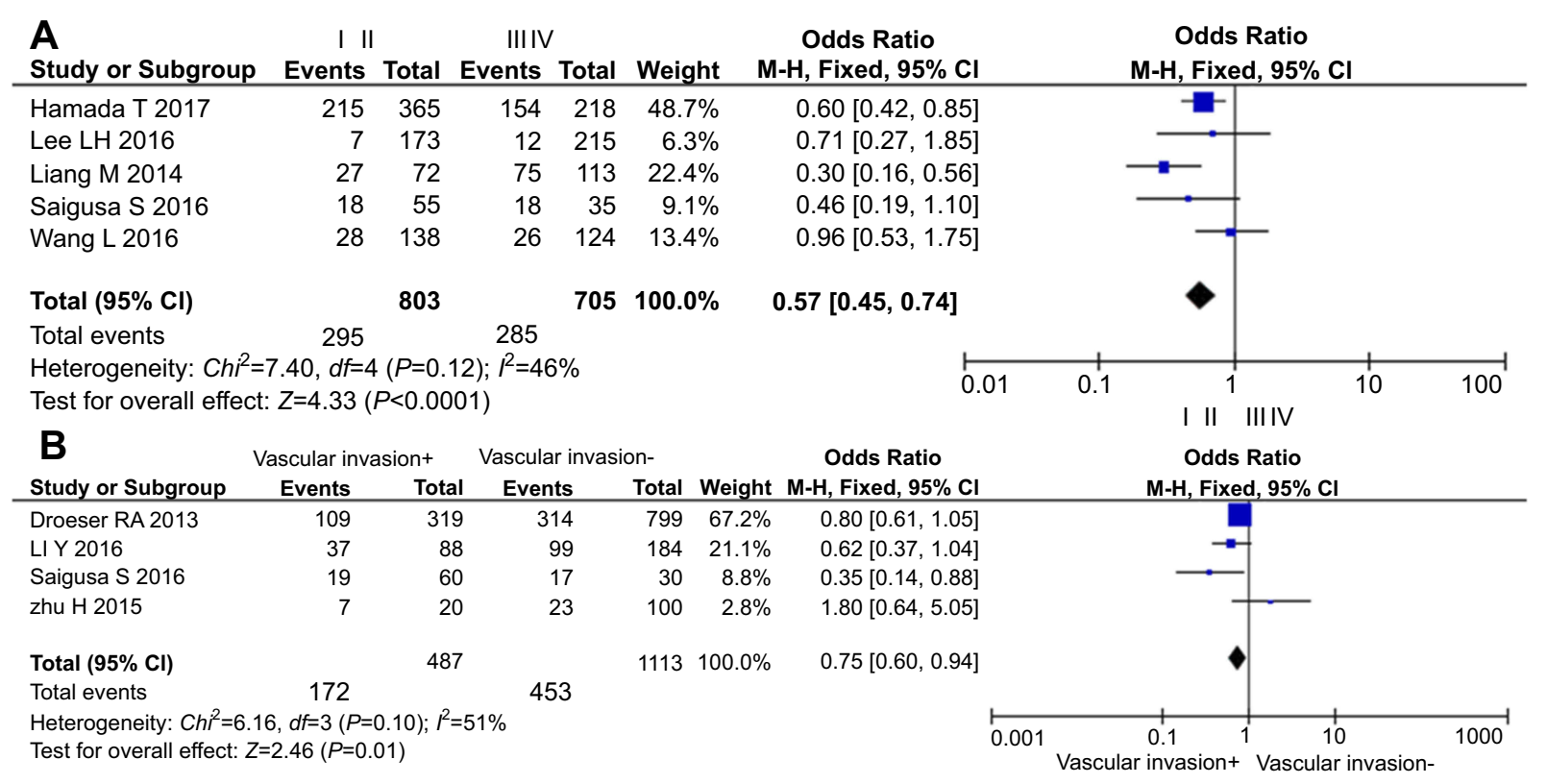

Figure 4 Forest plots for the association between PD-LI expression and clinicopathological parameters. (A) Tumor stage; (B) vascular invasion-negativity.

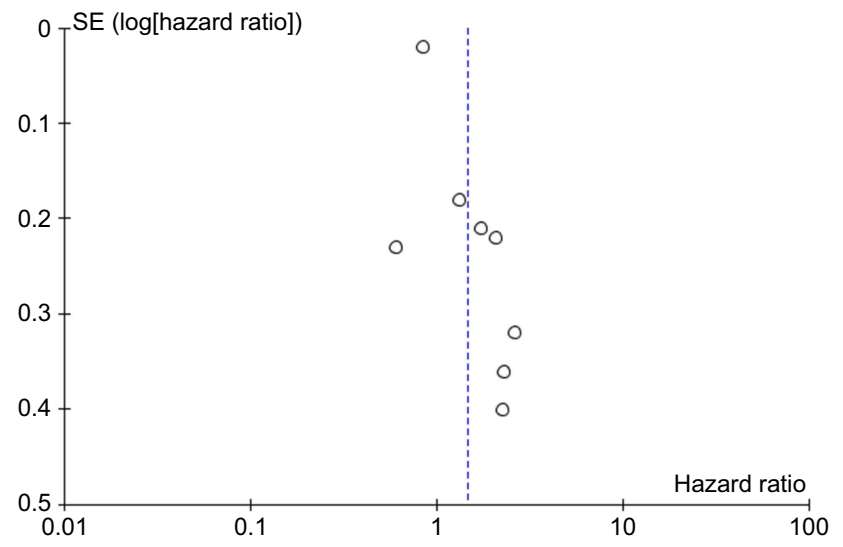

Figure 5 Funnel plot of publication bias for overall survival.

L1 expression and tumor stage, vascular invasion, MSI/ MMR mutation, etc. In our meta-analysis, we concentrate on the clinicopathological characteristics of patients with colorectal cancer and the prognostic validity of PD-L1 in colorectal cancer.

The interaction between PD-L1 and PD-1 can lead to exhausted phenotype and dysfunction of T-cells which protected tumor cells escape from the host immune surveillance. $^{40}$ Immune-checkpoint blockades become a new way in immunotherapy for cancer and it gradually changes the standard treatment of advanced-stage cancers. According to reports, overexpression of PD-1 and PD-L1 on tumor-infiltrating lymphocytes (TIL) and tumor cells (TC) correlates with poor prognosis in several cancers. ${ }^{41}$
Such as breast cancer, ${ }^{42}$ renal cell cancer, ${ }^{43}$ lung cancer, ${ }^{44}$ glioma. ${ }^{45}$ In colorectal cancer, this is in agreement with the study by Hamada et al, ${ }^{19}$ which indicated the association of PD-L1 overexpression and poor OS in CRCs (HR 1.33, 95\% CI 0.94-1.89). Additionally, Song et al, ${ }^{23}$ using tissue microarray immunohistochemistry to detect 404 CRC patient samples, univariate analysis disclosed that patients overexpression of PD-L1 had poorer overall survival $(p<0.001 ; \mathrm{HR}=2.07$ (1.342-3.193). But, multivariate analysis did not support PD-L1 as an independent prognostic factor $(p=0.548)$. Otherwise, some similar studies have revealed similar results. In contrast, a study of Droeser et $\mathrm{al}^{25}$ which involved 1420 CRCs patients, found the patients with MMR-proficient with PD-L1 overexpression correlated with better OS $(p=0.003$; HR $=0.84$ (0.79-0.88); In univariate analysis, PD-L1 overexpression in MMRproficient CRC was significantly correlated with early $\mathrm{T}$ stage, absence of lymph node metastases, lower tumor grade, absence of vascular invasion. Moreover, in the study by $\mathrm{Li}$ et al, ${ }^{26}$ this involved two cohorts; In the TCGA cohort, higher PD-L1 expressions indicated a better OS $(p=0.002)$. In FUSCC cohort, expressions of PD-L1 on tumor cells were associated with better OS $(p=0.002)$ and DFS ( $p=0.004)$ of CRC patients. Therefore, a meta-analysis of available data on the prognostic significance of PD-L1 will be useful and imperatively needed.

In this meta-analysis, we synthesized twelve pieces of research relevant to prognosis and clinicopathological of PD- 
L1 expression in colorectal cancer. As we know, this is the first meta-analysis focus on the prognosis and clinicopathological characteristics of patients with colorectal cancer. Our data indicated that PD-L1 overexpression was associated with shorter OS (HR 1.47, 95\% CI $=1.01-2.15, p=0.04$ ) and shorter RFS/DFS (HR 1.47, 95\% CI $=1.01-2.15$, $p=0.04$ ), suggesting that PD-L1 expression could serve as an important prognostic factor for colorectal cancer. Related research shows that immune evasion caused by PD-1/PD-L1 pathway may be related to poor infiltration of $\mathrm{T}$ cells into tumors and lack activation of cancer-specific $\mathrm{T}$ cells in the tumor microenvironment. ${ }^{46}$ Moreover, PD-L1 overexpression was increased in patients with inferior tumor stage $(\mathrm{OR}=0.57,95 \% \mathrm{CI}: 0.45,0.74, p<0.0001)$ and Vascular invasion-negativity $(\mathrm{OR}=0.75,95 \% \mathrm{CI}: 0.6,0.94, p=0.01)$. For the present study, we didn't find relationship between PD-L1 expression and age, gender, tumor location, tumor differentiation, $\mathrm{pT}$ stage, $\mathrm{pN}$ stage, MSI/MMR status.

There were several limitations to our study. Some significant heterogeneity can be observed in our study. These heterogeneities may have different sources. Firstly, only articles published in English were included in this metaanalysis. Secondly, the sample sizes of the studies enrolled in our analysis were comparatively small. Third, PD-L1 positivity was evaluated by using different antibody and dilution which affect the sensitivity of IHC. Fourth, the cut-off value where PD-L1 expressed was varied among the studies. So, future researches should make efforts in the standardization of PDL1 expression detection. Additionally, because not all study hasa distinction about MSI/MMR status and not provide sufficient information which may bring heterogeneity to the meta-analysis. Based on our observations, we believe that the results of our meta-analysis should be noticed and should be validated by additional studies with appropriate multivariate analyses.

Our investigation exhibited that the overexpression of PD-L1 indicates a poor prognosis and clinicopathological features and that treatment with anti-PD-L1 antibodies should be attempted in patients with CRCs patient in the future.

\section{Disclosure}

The authors report no conflicts of interest in this work.

\section{References}

1. Chen W, Zheng R, Baade PD, et al. Cancer statistics in China, 2015. CA Cancer J Clin. 2016;66(2):115-132. doi:10.3322/caac.21338
2. Siegel RL, Miller KD, Jemal A. Cancer statistics, 2017. CA Cancer J Clin. 2017;67(1):7-30. doi:10.3322/caac.21387

3. Siegel RL, Miller KD, Fedewa SA, et al. Colorectal cancer statistics, 2017. CA Cancer J Clin. 2017;67(3):177-193. doi:10.3322/caac.21395

4. Kuipers EJ, Grady WM, Lieberman D, et al. Colorectal cancer. Nat Rev Dis Primers. 2015;1:15065. doi:10.1038/nrdp.2015.65

5. Xiang B, Snook AE, Magee MS, Waldman SA. Colorectal cancer immunotherapy. Discov Med. 2013;15(84):301-308.

6. Hubbard JM, Grothey A. Colorectal cancer in 2014: progress in defining first-line and maintenance therapies. Nat Rev Clin Oncol. 2015;12(2):73-74. doi:10.1038/nrclinonc.2014.233

7. Fakih MG. Metastatic colorectal cancer: current state and future directions. J Clin oncol. 2015;33(16):1809-1824. doi:10.1200/ JCO.2014.59.7633

8. Dong H, Zhu G, Tamada K, Chen L. B7-H1, a third member of the B7 family, co-stimulates T-cell proliferation and interleukin-10 secretion. Nat Med. 1999;5(12):1365-1369. doi:10.1038/70932

9. Freeman GJ, Long AJ, Iwai Y, et al. Engagement of the PD-1 immunoinhibitory receptor by a novel B7 family member leads to negative regulation of lymphocyte activation. J Exp Med. 2000;192(7):1027-1034.

10. Topalian SL, Sznol M, McDermott DF, et al. Survival, durable tumor remission, and long-term safety in patients with advanced melanoma receiving nivolumab. $J$ Clin oncol. 2014;32(10):1020-1030. doi:10.1200/JCO.2013.53.0105

11. Curiel TJ, Wei S, Dong H, et al. Blockade of B7-H1 improves myeloid dendritic cell-mediated antitumor immunity. Nat Med. 2003;9(5):562-567. doi:10.1038/nm863

12. Perrot I, Blanchard D, Freymond N, et al. Dendritic cells infiltrating human non-small cell lung cancer are blocked at immature stage. J Immunol. 2007;178(5):2763-2769.

13. Wu K, Kryczek I, Chen L, Zou W, Welling TH. Kupffer cell suppression of CD8+ T cells in human hepatocellular carcinoma is mediated by B7-H1/programmed death-1 interactions. Cancer Res. 2009;69 (20):8067-8075. doi:10.1158/0008-5472.CAN-09-0901

14. Kuang DM, Zhao Q, Peng C, et al. Activated monocytes in peritumoral stroma of hepatocellular carcinoma foster immune privilege and disease progression through PD-L1. J Exp Med. 2009;206 (6):1327-1337. doi:10.1084/jem.20082173

15. Nazareth MR, Broderick L, Simpson-Abelson MR, Kelleher RJ Jr., Yokota SJ, Bankert RB. Characterization of human lung tumor-associated fibroblasts and their ability to modulate the activation of tumor-associated T cells. J Immunol. 2007;178(9):5552-5562.

16. Ghebeh H, Mohammed S, Al-Omair A, et al. The B7-H1 (PD-L1) T lymphocyte-inhibitory molecule is expressed in breast cancer patients with infiltrating ductal carcinoma: correlation with important high-risk prognostic factors. Neoplasia (New York, NY). 2006;8 (3):190-198. doi:10.1593/neo.05733

17. Denkert C, von Minckwitz G, Brase JC, et al. Tumor-infiltrating lymphocytes and response to neoadjuvant chemotherapy with or without carboplatin in human epidermal growth factor receptor 2-positive and triple-negative primary breast cancers. J Clin oncol. 2015;33(9):983-991. doi:10.1200/JCO.2014.58.1967

18. Chen L, Han X. Anti-PD-1/PD-L1 therapy of human cancer: past, present, and future. J Clin Invest. 2015;125(9):3384-3391. doi:10.1172/JCI80011

19. Hamada T, Cao Y, Qian ZR, et al. Aspirin use and colorectal cancer survival according to tumor CD274 (programmed cell death 1 ligand 1) expression status. J Clin oncol. 2017;35(16):1836-1844. doi:10.1200/JCO.2016.70.7547

20. Liang M, Li J, Wang D, et al. T-cell infiltration and expressions of $\mathrm{T}$ lymphocyte co-inhibitory $\mathrm{B} 7-\mathrm{H} 1$ and $\mathrm{B} 7-\mathrm{H} 4$ molecules among colorectal cancer patients in northeast China's Heilongjiang province. Tumour Biol. 2014;35(1):55-60. doi:10.1007/s13277-013-1006-6

21. Saigusa S, Toiyama Y, Tanaka K, et al. Implication of programmed cell death ligand 1 expression in tumor recurrence and prognosis in rectal cancer with neoadjuvant chemoradiotherapy. Int J Clin Oncol. 2016;21(5):946-952. doi:10.1007/s10147-016-0962-4 
22. Shi SJ, Wang LJ, Wang GD, et al. B7-H1 expression is associated with poor prognosis in colorectal carcinoma and regulates the proliferation and invasion of HCT116 colorectal cancer cells. PLoS One. 2013;8(10):e76012. doi:10.1371/journal.pone.0076012

23. Song M, Chen D, Lu B, et al. PTEN loss increases PD-L1 protein expression and affects the correlation between PD-L1 expression and clinical parameters in colorectal cancer. PLoS One. 2013;8(6): e65821. doi:10.1371/journal.pone.0065821

24. Zhu H, Qin H, Huang Z, et al. Clinical significance of programmed death ligand-1 (PD-L1) in colorectal serrated adenocarcinoma. Int J Clin Exp Pathol. 2015;8(8):9351-9359.

25. Droeser RA, Hirt C, Viehl CT, et al. Clinical impact of programmed cell death ligand 1 expression in colorectal cancer. Eur J Cancer. 2013;49(9):2233-2242. doi:10.1016/j.ejca.2013.02.015

26. Li Y, Liang L, Dai W, et al. Prognostic impact of programed cell death-1 (PD-1) and PD-ligand 1 (PD-L1) expression in cancer cells and tumor infiltrating lymphocytes in colorectal cancer. Mol Cancer. 2016;15(1):55. doi:10.1186/s12943-016-0539-x

27. Wu P, Wu D, Li L, Chai Y, Huang J, Soutto M. PD-L1 and survival in solid tumors: a meta-analysis. PLoS One. 2015;10(6):e0131403. doi:10.1371/journal.pone.0131403

28. Pyo JS, Kang G, Kim JY. Prognostic role of PD-L1 in malignant solid tumors: a meta-analysis. Int J Biol Markers. 2017;32(1):e68e74. doi:10.5301/jbm.5000225

29. Xiang X, Yu PC, Long D, et al. Prognostic value of PD -L1 expression in patients with primary solid tumors. Oncotarget. 2018;9 (4):5058-5072. doi:10.18632/oncotarget.23580

30. Moher D, Liberati A, Tetzlaff J, Altman DG. Preferred reporting items for systematic reviews and meta-analyses: the PRISMA statement. BMJ. 2009;339:b2535. doi:10.1136/bmj.b2651

31. Koganemaru S, Inoshita N, Miura Y, et al. Prognostic value of programmed death-ligand 1 expression in patients with stage III colorectal cancer. Cancer Sci. 2017;108(5):853-858. doi:10.1111/cas.13229

32. Wang L, Liu Z, Fisher KW, et al. Prognostic value of programmed death ligand 1, p53, and Ki-67 in patients with advanced-stage colorectal cancer. Hum Pathol. 2018;71:20-29. doi:10.1016/j.humpath.2017.07.014

33. Lee LH, Cavalcanti MS, Segal NH, et al. Patterns and prognostic relevance of PD-1 and PD-L1 expression in colorectal carcinoma. Mod Pathol. 2016;29(11):1433-1442. doi:10.1038/modpathol.2016.139

34. Wang L, Ren F, Wang Q, et al. Significance of programmed death ligand 1 (PD-L1) immunohistochemical expression in colorectal cancer. Mol Diagn Ther. 2016;20(2):175-181. doi:10.1007/s40291016-0188-1
35. Topalian SL, Hodi FS, Brahmer JR, et al. Safety, activity, and immune correlates of anti-PD-1 antibody in cancer. $N$ Engl J Med. 2012;366(26):2443-2454. doi:10.1056/NEJMoa1200690

36. Rizvi NA, Mazieres J, Planchard D, et al. Activity and safety of nivolumab, an anti-PD-1 immune checkpoint inhibitor, for patients with advanced, refractory squamous non-small-cell lung cancer (CheckMate 063): a phase 2, single-arm trial. Lancet Oncol. 2015;16(3):257-265. doi:10.1016/S1470-2045(15)70054-9

37. Ansell SM, Lesokhin AM, Borrello I, et al. PD-1 blockade with nivolumab in relapsed or refractory Hodgkin's lymphoma. $N$ Engl J Med. 2015;372(4):311-319. doi:10.1056/NEJMoa1411087

38. Hamid O, Robert C, Daud A, et al. Safety and tumor responses with lambrolizumab (anti-PD-1) in melanoma. $N$ Engl J Med. 2013;369 (2):134-144. doi:10.1056/NEJMoa1305133

39. Seiwert TY, Burtness B, Mehra R, et al. Safety and clinical activity of pembrolizumab for treatment of recurrent or metastatic squamous cell carcinoma of the head and neck (KEYNOTE-012): an open-label, multicentre, phase 1b trial. Lancet Oncol. 2016;17(7):956-965. doi:10.1016/S1470-2045(16)30066-3

40. Ohaegbulam KC, Assal A, Lazar-Molnar E, Yao Y, Zang X. Human cancer immunotherapy with antibodies to the PD-1 and PD-L1 pathway. Trends Mol Med. 2015;21(1):24-33. doi:10.1016/j. molmed.2014.10.009

41. Meng X, Huang Z, Teng F, Xing L, Yu J. Predictive biomarkers in PD-1/PD-L1 checkpoint blockade immunotherapy. Cancer Treat Rev. 2015;41(10):868-876. doi:10.1016/j.ctrv.2015.11.001

42. Guo Y, Yu P, Liu Z, et al. Prognostic and clinicopathological value of programmed death ligand-1 in breast cancer: a meta-analysis. PLoS One. 2016;11(5):e0156323.

43. Xu F, Xu L, Wang Q, An G, Feng G, Liu F. Clinicopathological and prognostic value of programmed death ligand-1 (PD-L1) in renal cell carcinoma: a meta-analysis. Int $J$ Clin Exp Med. 2015;8 (9):14595-14603.

44. Aguiar PN Jr., Santoro IL, Tadokoro H, et al. The role of PD-L1 expression as a predictive biomarker in advanced non-small-cell lung cancer: a network meta-analysis. Immunotherapy. 2016;8 (4):479-488. doi:10.2217/imt-2015-0002

45. Xue S, Song G, Yu J. The prognostic significance of PD-L1 expression in patients with glioma: a meta-analysis. Sci Rep. 2017;7 (1):4231. doi:10.1038/s41598-017-04023-x

46. Kim JM, Chen DS. Immune escape to PD-L1/PD-1 blockade: seven steps to success (or failure). Ann Oncol. 2016;27(8):1492-1504. doi:10.1093/annonc/mdw217 


\section{Supplementary material}

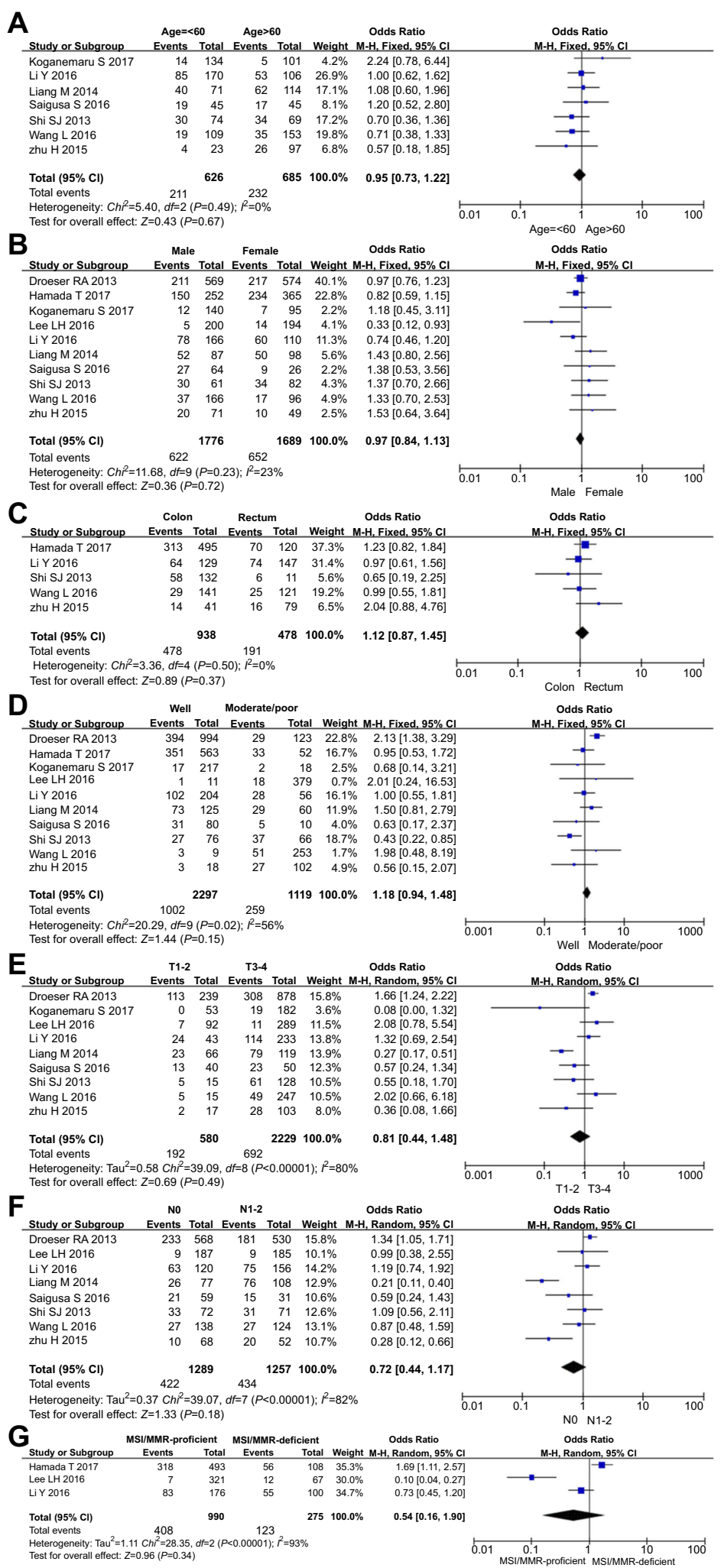

Figure SI Forest plots for the association between PD-LI expression and clinicopathological parameters. (A) Age; (B) sex; (C) tumor location; (D) tumor differentiation; (E) PT stage; (F) pN stage; and (G) MSI/MMR status. 


\section{Publish your work in this journal}

OncoTargets and Therapy is an international, peer-reviewed, open access journal focusing on the pathological basis of all cancers, potential targets for therapy and treatment protocols employed to improve the management of cancer patients. The journal also focuses on the impact of management programs and new therapeutic agents and protocols on patient perspectives such as quality of life, adherence and satisfaction. The manuscript management system is completely online and includes a very quick and fair peer-review system, which is all easy to use. Visit http://www.dovepress.com/ testimonials.php to read real quotes from published authors. 